Check for updates

Cite this: RSC Adv., 2018, 8, 34682

\title{
Novel hard carbon/graphite composites synthesized by a facile in situ anchoring method as high-performance anodes for lithium-ion batteries $\dagger$
}

\author{
Chuanzhang Ge, (D)*ab Zhenghua Fan, ${ }^{\text {ab }}$ Jie Zhang, ${ }^{\text {ab }}$ Yongmin Qiao, ${ }^{\text {ab }}$ \\ Jianming Wang (D) ${ }^{c}$ and Licheng Ling ${ }^{d}$
}

In this report, novel hard carbon/graphite composites are prepared by a simple in situ particle anchoring method, followed by carbonization. The effects of loading content of hard carbon on the structure and electrochemical performance of the composites are investigated. The SEM results show that the hard carbon particles are anchored randomly on the surface of graphite. The electrochemical measurements demonstrate that an appropriate loading content of hard carbon can remarkably increase the specific reversible capacity of graphite, which is mainly contributed by lithiation in hard carbon, whereas excessive loading leads to the formation of a thick particle shell onto the surface of graphite, which deteriorates the initial coulombic efficiency drastically. Kinetic tests further show that excessive loading of hard carbon is unfavorable for lithium-ion diffusion probably due to the increased interface distance and decreased electroconductivity. The composite loaded with 10 wt\% hard carbon exhibits balanced lithium storage performance with high reversible capacity of $366 \mathrm{~mA} \mathrm{~h} \mathrm{~g}^{-1}$, high initial coulombic efficiency ( 91.3\%), and superior rate capability and cycling performance. Thus, in this study, we suggest a facile and effective strategy to fabricate a promising graphite anode material for high-performance lithium-ion batteries.

Received 28th August 2018 Accepted 23rd September 2018 DOI: $10.1039 / \mathrm{c} 8 \mathrm{ra07170e}$

rsc.li/rsc-advances impede its further development in the next-generation LIBs with high energy and high power density. Therefore, intense efforts are needed to find new ways of improving the performance of this commercialized anode material.

To meet the increasing need for high-performance graphite, various materials, such as carbonaceous materials (soft carbon and hard carbon), ${ }^{5-7}$ ion-functional groups, ${ }^{8}$ silicons, ${ }^{9-11}$ and metal compounds, ${ }^{12,13}$ have been coated onto the graphite surface to suppress the irreversible solvated lithium intercalation into graphite layers as well as to improve the rate capability. Among these materials, hard carbon-based materials have been proven to be the most effective modifiers for graphite due to their highly disordered and porous structure, which can not only shorten the transport distance for $\mathrm{Li}^{+}$but also offer large electrode-electrolyte interface for charge-transfer reaction. ${ }^{\mathbf{1 4}}$ Generally, hard carbon is composited to graphite by directly mixing or through surface coating by pyrolyzing adequate precursors in the practical production. ${ }^{15,16}$ However, the traditional coating method has the limitation of low carbon loading, ${ }^{17}$ as high carbon loading can easily cause serious particle agglomeration, thus resulting in low production yield. While direct mixing of graphite and hard carbon can cause phase separation due to different true densities and tap densities. Thus, scalable preparation of low-cost hard carbon/ graphite composites with high hard carbon ratio, controllable 
structure and excellent lithium storage performance is still a challenge.

In this study, a facile method is reported for large-scale fabrication of a novel hard carbon/graphite composite with unique morphology by in situ anchoring of bare graphite with hard carbon particles of smaller size. To achieve this goal, a lowcost pitch with high softening point was used as the precursor for the production of hard carbon through surface solidification by phosphoric anhydride and carbonization. The microstructures of the obtained composites were characterized, and the corresponding anode performances were investigated and optimized. The results showed that the composite with an optimal loading of hard carbon displays promising electrochemical performance with high reversible capacity, good rate capability, and desirable cycling stability.

\section{Experimental}

\subsection{Material preparation}

For the purpose of this study, artificial graphite (AG, Shanghai Shanshan Tech Co., Ltd.) with $D_{50}$ size of about $18.6 \mu \mathrm{m}$ was used as the raw material. An isotropic petroleum pitch (Dalian Mingqiang Chemical Materials Co., Ltd., China) with a softening point of $250{ }^{\circ} \mathrm{C}$ was used as the carbon source for developing hard carbon. The pitch was milled and classified to adjust the median diameter $D_{50}$ to $c a$. 3-4 $\mu \mathrm{m}$ before usage. Then, the hard carbon/graphite ( $\mathrm{HC} / \mathrm{G})$ composite was synthesized by the following procedure: $A G$ and pitch were first dispersed in distilled water to form a homogeneous suspension with total solid content of $50 \%$. The ratio of the pitch was in the range of 10-30 wt \% against the amount of AG. Then, the oxidizing agent $\mathrm{P}_{2} \mathrm{O}_{5}$ (PO) was added into the above suspension under vigorous stirring at room temperature to reach a concentration of $20 \mathrm{wt} \%$ (against the pitch). After that, the obtained slurry was oxidized and dried at $100{ }^{\circ} \mathrm{C}$ for $12 \mathrm{~h}$ in an oven to avoid pitch remelting during the following carbonization process. Thus, the obtained mixture was carbonized at $1000{ }^{\circ} \mathrm{C}$ for $2 \mathrm{~h}$ under flowing argon in a tube furnace at a heating rate of $5{ }^{\circ} \mathrm{C} \mathrm{min}^{-1}$. The resulting samples were noted as $\mathrm{HC} / \mathrm{G}-10$ and $\mathrm{HC} / \mathrm{G}-30$ according to the amount of pitch used. Before use, AG was also annealed at $1000{ }^{\circ} \mathrm{C}$. The schematic diagram of the preparation process for $\mathrm{HC} / \mathrm{G}$ is illustrated in Fig. 1.

\subsection{Material characterization}

The surface groups of the samples were determined by XPS with a Thermo K-Alpha+ system and a monochromatic Al-K $\alpha$ X-ray source. The spectra were analyzed and processed by using the XPSPEAK software (version 4.1). Peaks were deconvoluted using mixed Gauss-Lorentz sum function line shape and a Shirleytype background line. The morphology of the samples was obtained using scanning electron microscopy (SEM, FEI Quanta200). Particle size distribution of the prepared samples was determined with Malvern 2000 Instruments. The crystal phase composition of the obtained samples was characterized by X-ray diffraction (XRD) analysis using a Bruker D8 Advance diffractometer with $\mathrm{Cu} \mathrm{K}_{\alpha}$ radiation $(\lambda=0.1 .54056 \mathrm{~nm})$. Data were collected for scattering angles $(2 \theta)$ from 10 to $80^{\circ}$ with a scan rate of $2^{\circ} \mathrm{min}^{-1}$. The results of XRD analysis were processed using the Bragg and Scherrer equations. Raman spectra were recorded by a Raman spectrometer system (inVia-Reflex) using a laser with $532 \mathrm{~nm}$ excitation at room temperature. Nitrogen adsorption and desorption isotherms were measured using an automatic specific surface area of microporous/mesoporous physical adsorption analyzer (ASAP 2460 V2.01) at $77 \mathrm{~K}$. Surface areas were determined by the BET method.

\subsection{Electrochemical measurements}

The working electrodes were prepared by coating a mixture of the as-prepared material with Super $\mathrm{P}$ conductive agent and poly (vinylidene fluoride) (PVDF) binder at a weight ratio of 91.6 : 6.6 : 1.8 dissolved in $N$-methyl-2-pyrrolidone (NMP) on a copper foil. Electrochemical tests were carried out using CR2430 with a lithium metal foil as the counter electrode. To form the anode, the mixed slurry was coated on a copper foil, dried at $120{ }^{\circ} \mathrm{C}$ under vacuum for $12 \mathrm{~h}$, pressed using a roll-type mill under $100 \mathrm{MPa}$ of pressure, cut into discs $(16 \mathrm{~mm}$ in diameter), and then weighed using an ultra-fine balance to adjust the amount of active material. Coin-type cells were assembled in an Ar-filled glove box using a polyethylene film as a separator and lithium hexafluorophosphate $\left(\mathrm{LiPF}_{6}\right)$ solution (1 M) in a mixture of ethylene carbonate/diethyl carbonate/ethyl methyl carbonate (EC/DEC/EMC, $1: 1: 1$, v/v/v, Shanshan Tech Co., Ltd) as an electrolyte. Galvanostatic charge/discharge measurements were carried out using a Land Battery

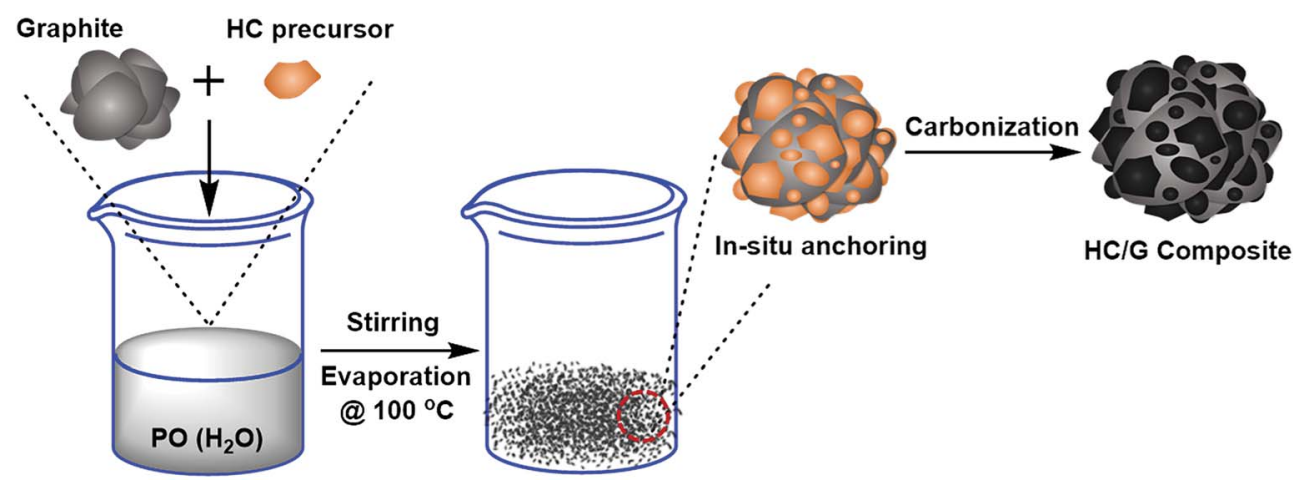

Fig. 1 Schematic presentation of the process for $\mathrm{HC} / \mathrm{G}$ composites. 
Measurement System (LAND CT2001A, China) in a fixed voltage range of $0-2.5 \mathrm{~V} v s$. $\mathrm{Li} / \mathrm{Li}^{+}$at room temperature. Cyclic voltammetry (CV) and electrochemical impedance spectroscopy (EIS) measurements were performed using an electrochemical working station (Zahner IM6ex) at room temperature. All the electrochemical tests were carried out at ambient temperature.

\section{Results and discussion}

\subsection{Physical properties of the HC/G composites}

HC/G composites are successfully prepared by co-blending AG, pitch powder and oxidizing agent PO in water, followed by successive drying and carbonization. Generally, the PO converts to $\mathrm{H}_{3} \mathrm{PO}_{4}$ firstly after dissolving in water and then connects with the superficial pitch molecules through an addition reaction during the drying process, by which the $\mathrm{P}=\mathrm{O}$ bonds are opened and new phosphorus-containing groups of $\mathrm{CO}-\mathrm{P}(\mathrm{O})(\mathrm{OH})_{2}$ are formed. Such phosphorus-containing hydroxyl groups extensively condense with other pitch molecules by releasing $\mathrm{H}_{2} \mathrm{O}$ and forming intermolecular cross-linked structures and thus realize the in situ solidification of the pitch precursor. ${ }^{18}$ During the high-temperature carbonization process, the released volatiles make the pitch particles stably cohere to the surface of AG. The pyrolysis behaviour of the samples after drying is first investigated by thermogravimetric analysis, as shown in Fig. S1. $\dagger$ Compared with the results for pristine AG, the first remarkable weight loss stages at $400{ }^{\circ} \mathrm{C}$ (for $\mathrm{HC} / \mathrm{G}-3$ ) and at $450{ }^{\circ} \mathrm{C}$ (for $\mathrm{HC} / \mathrm{G}-1$ ) are mainly due to multiple and complex reactions (cracking, polymerization, condensation, etc.) of lowmolecular-weight compounds in the pitch. ${ }^{19}$ The second weight loss stage at about $850{ }^{\circ} \mathrm{C}$ can be mainly due to the decomposition of some aromatic rings and escape of small phosphorus-containing groups.

To confirm the existence of phosphorus and its chemical bonding state in the carbonized samples, XPS analysis is also conducted, as shown in Fig. 2a. Compared with the results for AG (Fig. 2b), P 2p peaks are detected in XPS of HC/G-1 (Fig. 2c)
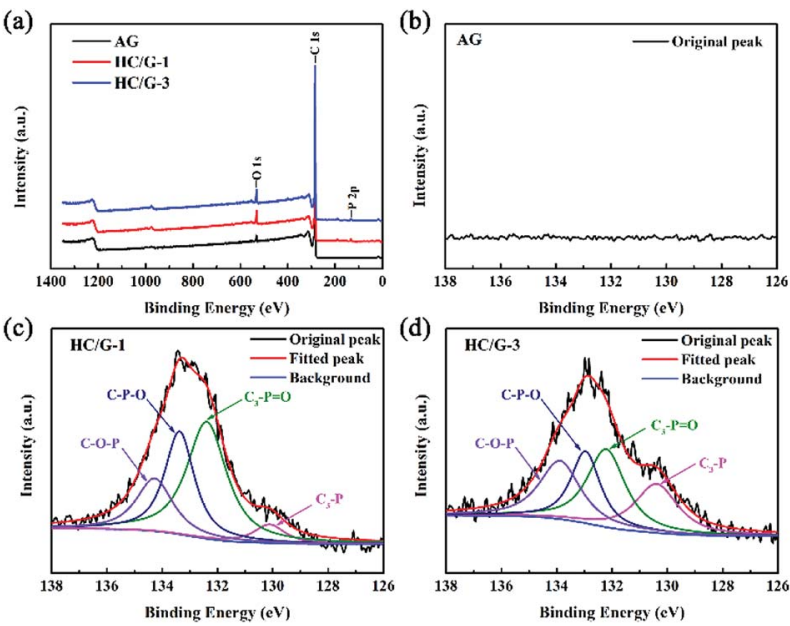

Fig. 2 (a) XPS spectra of the samples; (b) P 2p peak of AG; (c) P 2p peak of $\mathrm{HC} / \mathrm{G}-1$; (d) $\mathrm{P} 2 \mathrm{p}$ peak of HC/G-3. and HC/G-3 (Fig. 2d), indicating the existence of phosphorus functional groups in the obtained composites. The bonding energies of $\mathrm{P} 2 \mathrm{p}$ at $133.9 \mathrm{eV}, 133.0 \mathrm{eV}, 132.2 \mathrm{eV}$ and $130.4 \mathrm{eV}$ are assigned to the linkages of $\mathrm{C}-\mathrm{O}-\mathrm{P}, \mathrm{C}-\mathrm{P}-\mathrm{O}, \mathrm{C}_{3}-\mathrm{P}=\mathrm{O}$ and $\mathrm{C}_{3}-\mathrm{P}$, respectively, ${ }^{18}$ indicating strong evolution of the phosphorus structures during the high-temperature treatment. The presence of $\mathrm{C}-\mathrm{P}$ bonds is a result of an effective bonding reaction between the pitch precursor and phosphoric acid on a molecular scale. In addition, $\mathrm{O}$ 1s spectra are also analysed in Fig. S2. $\dagger$ Based on calculations, the relative contents of phosphorus and oxygen species are summarized in Table S2. $\dagger$ It can be found that the content of $\mathrm{P}$ and $\mathrm{O}$-containing groups varies with the increase in the pitch precursor and PO.

Fig. 3 displays the SEM images of pristine AG and the obtained composite HC/Gs. As can be seen, AG presents (Fig. 3a and b) an original irregular multi-particle morphology with smooth surface. After the in situ anchoring process, the morphology of obtained HC/G-1 (Fig. 3c and d) does not change appreciably as compared to that of the original one except that many hard carbon particles (marked with red arrows) are homogeneously present on the surface and gaps of AG. However, the surface of this composite appears to be rough, especially when the amount of anchored hard carbon increases (HC/G-3), as shown in Fig. 3e and f. The in situ-anchored hard carbon particles exhibit sharp-edged morphologies, which can be the main reason for the rough appearance. Such a unique structure might be beneficial to reduce the electrolyte sensitivity of the inner graphite..$^{\mathbf{2 0 1} 21}$

Moreover, the SEM mapping results in Fig. S3† further verify the existence and uniform distribution of elemental $\mathrm{P}$ and $\mathrm{O}$ on the surface of the composites. No clear agglomeration of the graphite particles is found after the carbonization process, suggesting that the pitch precursor has been well-oxidized

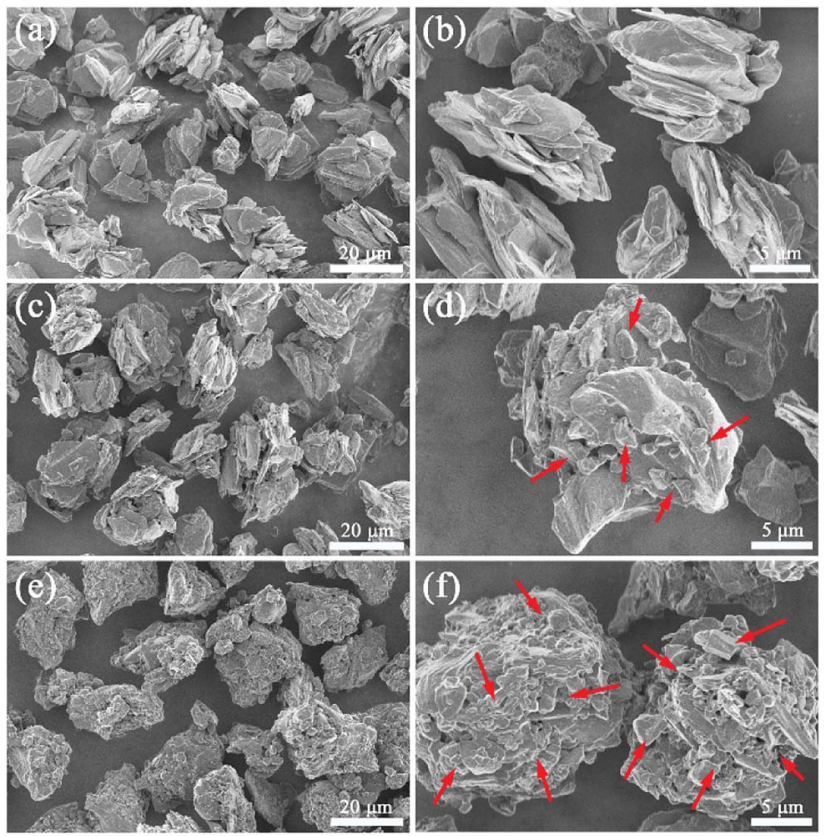

Fig. 3 SEM images of sample (a) and (b) AG, (c) and (d) HC/G-1 and (e) and (f) HC/G-3. 

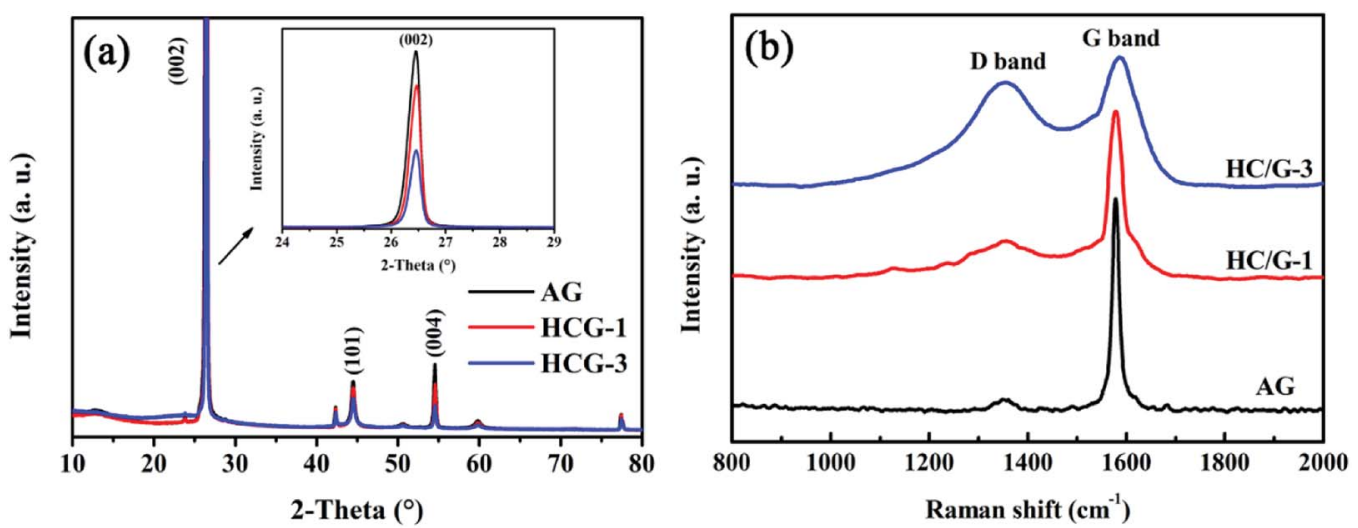

Fig. 4 XRD patterns (a) and Raman spectra (b) of AG, HC/G-1 and HC/G-3.

under low temperature by PO. Such results can be further demonstrated by the particle size distributions presented in Fig. S4. $\dagger$ The average particle diameters $\left(D_{50}\right)$ of AG, HC/G-1 and HC/G-3 are measured to be 18.6, 19.3, $20.4 \mu \mathrm{m}$, respectively, suggesting that the pitch based on hard carbon particles is adhered to the surface of the graphite. The nanostructure of the pitch based on hard carbon is further characterized by TEM (Fig. S5 $\dagger$ ). The obtained TEM images reveal a typical turbostratic structure of the sample, which matches the "house of cards" model for hard carbons. ${ }^{22}$

X-ray diffraction (XRD) patterns are obtained to further confirm the structural features of the obtained samples. As shown in Fig. 4a, both original AG and HC/G composites show sharp diffraction peaks at $2 \theta$ of around $26^{\circ}, 44^{\circ}$ and $54^{\circ}$, which are ascribed to the (002), (101) and (004) planes in graphitic carbon, ${ }^{23}$ respectively. The diffraction peak intensities of HC/G1 and HC/G-3 decrease with the increase in hard carbon loading, indicating that a well-designed disordered carbon layer is formed on the graphite surface. ${ }^{24}$ No peak that belongs to any impurity or secondary phase is detected in the XRD patterns, suggesting that the used oxidizing agent PO is homogenously consumed in the final composites after carbonization. Based on the Bragg equation, ${ }^{25}$ the interlayer space $d_{(002)}$ values are calculated to be 0.3368 and $0.3370 \mathrm{~nm}$ (Table 1) for HC/G-1 and HC/G-3, respectively, which are larger than that of original AG $\left(d_{(002)}=0.3358 \mathrm{~nm}\right)$. Moreover, the average crystal sizes $L_{\mathrm{c}}$ and $L_{\mathrm{a}}$ both decrease with the increase in hard carbon loading. The powder resistivities of AG and $\mathrm{HC} / \mathrm{G}$ composites are also estimated and listed in Table 1. As can be seen, the powder

Table 1 Structure parameters of the three samples

\begin{tabular}{|c|c|c|c|c|c|c|}
\hline & $d_{002}$ & $L_{\mathrm{c}}$ & $L_{\mathrm{a}}$ & & $\mathrm{PR}^{b}$ & $S_{\mathrm{BET}}{ }^{c}$ \\
\hline Samples & $(\mathrm{nm})$ & $(\mathrm{nm})$ & $(\mathrm{nm})$ & $I_{\mathrm{D}} / I_{\mathrm{G}}{ }^{a}$ & $\Omega \mathrm{mm}$ & $\left(\mathrm{m}^{2} \mathrm{~g}^{-1}\right)$ \\
\hline AG & 0.3358 & 758 & 2426 & 0.10 & 0.25 & 1.8 \\
\hline HC/G-1 & 0.3368 & 734 & 2355 & 0.28 & 0.37 & 1.6 \\
\hline $\mathrm{HC} / \mathrm{G}-3$ & 0.3370 & 664 & 1839 & 0.83 & 0.44 & 1.2 \\
\hline
\end{tabular}

${ }^{a}$ The integral intensity ratio of D-peak against G-peak. ${ }^{b}$ Powder resistivity. ${ }^{c}$ BET specific surface area. resistivity increases significantly with the increase in $\mathrm{HC}$ content from $0.25 \mathrm{~m} \Omega \mathrm{mm}(\mathrm{AG})$ to $0.37 \mathrm{~m} \Omega \mathrm{mm}(\mathrm{HC} / \mathrm{G}-1)$ and then to $0.44 \mathrm{~m} \Omega \mathrm{mm}(\mathrm{HC} / \mathrm{G}-3)$. This considerable increase in powder resistivity is due to the low conductivity of the introduced hard carbon in the HC/G composites.

Raman spectra are also obtained to further probe the microstructure of the obtained composites. As shown in Fig. 4b, all the Raman spectra show clear D-band at $\sim 1360 \mathrm{~cm}^{-1}$ and Gband peak at $\sim 1580 \mathrm{~cm}^{-1}$, corresponding to the disordered $\mathrm{sp}^{2}$ carbons and $\mathrm{E}_{2 \mathrm{~g}}$ mode (in-plane stretching vibration) graphitic carbons. ${ }^{26,27}$ The integral intensity ratio of $\mathrm{D}$ to $\mathrm{G}$ band, $I_{\mathrm{D}} / I_{\mathrm{G}}$, is well-established as an indicator of crystallinity for the local carbon in carbon materials. ${ }^{28}$ The numerical values of $I_{\mathrm{D}} / I_{\mathrm{G}}$ are calculated to be $0.10,0.28$ and 0.83 for AG, HC/G-1 and HC/G-3, respectively (Table 1 ). Clearly, the $I_{\mathrm{D}} / I_{\mathrm{G}}$ value increases with the increase in loaded hard carbon, further suggesting the increase in defects and the growth of local disordered structures in the composites. This result agrees well with the XRD data illustrated in Fig. 4a.

Fig. 5 shows the nitrogen adsorption-desorption isotherms of the obtained composites. It can be found that a typical IV isotherm with a distinct hysteresis loop at relative pressure $P / P_{0}$ between 0.45 and 0.95 is observed for original AG, indicating

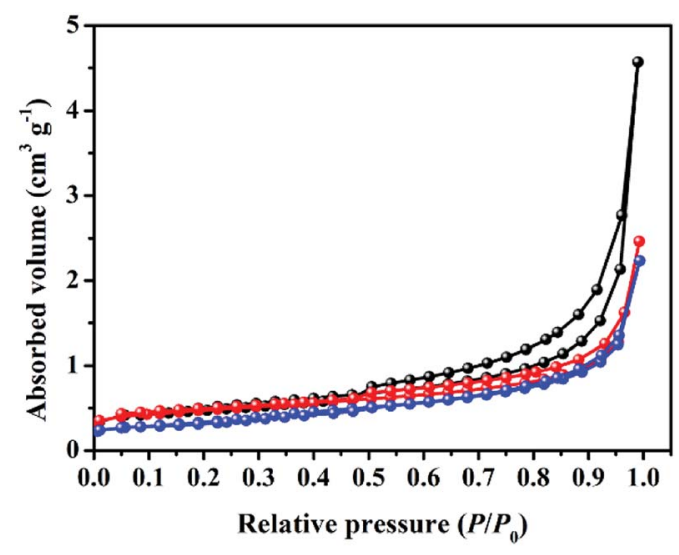

Fig. $5 \mathrm{~N}_{2}$ absorption/desorption isotherms of the samples. 
the presence of some open mesopores. ${ }^{29}$ However, the hysteresis loop gradually shrinks with the increase in hard carbon loading, suggesting clear change in nanopores of the composites after the anchoring process. Especially for sample HC/G-3, the hysteresis loop nearly disappears, and the total nitrogen absorption volume decreases to the minimum. The specific surface areas $\left(S_{\mathrm{BET}}\right)$ of $\mathrm{AG}, \mathrm{HC} / \mathrm{G}-1$ and $\mathrm{HC} / \mathrm{G}-3$ are calculated to be $1.8,1.6$ and $1.2 \mathrm{~m}^{2} \mathrm{~g}^{-1}$ (Table 1 ), respectively. Such low $S_{\text {BET }}$ values indicate that the nanopores are closed or that their entrances are smaller than those of the nitrogen molecules. Combined with SEM results (Fig. 3), the gradual decrease in $S_{\mathrm{BET}}$ is possibly caused by the thermal deposition of the volatiles (released from the oxidized pitch) into the defective nanopores, which acts as a pore healing process during carbonization. Additionally, the increased quantity of doped PO within the composite also has strong effect on the carbon structure, which might destroy the pores. ${ }^{30}$

\subsection{Electrochemical properties of the $\mathrm{HC} / \mathrm{G}$ composites}

The electrochemical properties are further investigated by cointype half-cells (CR2430 R-type) to understand the $\mathrm{Li}^{+}$storage behavior in the obtained samples. Fig. 6 shows the cyclic voltammetry (CV) curves of the samples for the first three cycles from 0 to $2.5 \mathrm{~V}$ at a scanning rate of $0.5 \mathrm{mV} \mathrm{s}^{-1}$. Clearly, two stages of lithium-ion intercalation/deintercalation occurring below $1.3 \mathrm{~V}$ can be observed during scanning. Generally, the reduction peak at about $0.01 \mathrm{~V}$ is assigned to the distinct lithiation process, whereas the oxidation peak around $0.38 \mathrm{~V}$ corresponds to delithiation from graphitic structure. To be noted, the small reduction peak at around $0.57 \mathrm{~V}$ in the first cathodic scan is ascribed to the formation of a solid electrolyte interphase (SEI) layer, ${ }^{31}$ which causes major irreversible capacities in the first cycle. Compared with the results for pristine AG, the intersection of oxidation and reduction curves for the composites increases from $c a$. $0.8 \mathrm{~V}$ to $c a$. $1.3 \mathrm{~V}$, thus

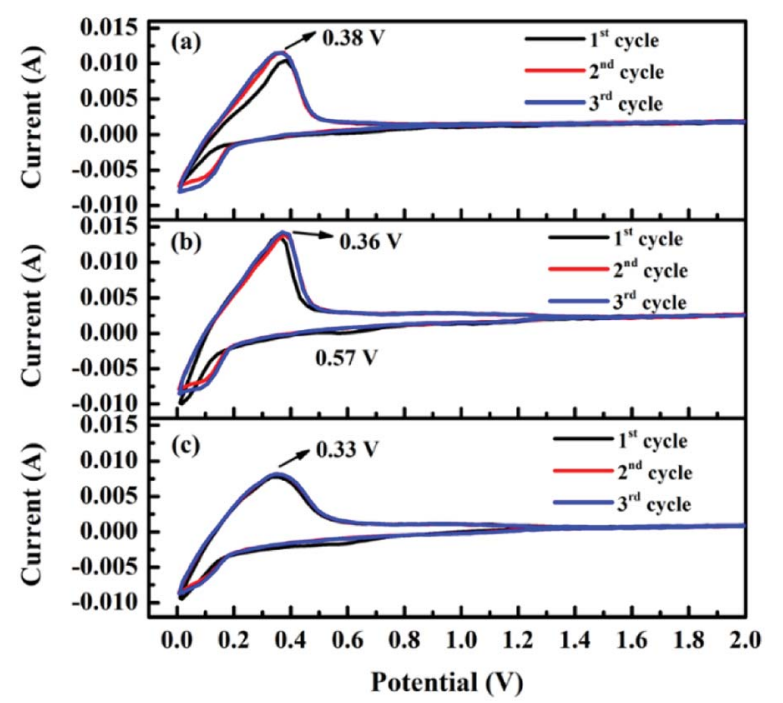

Fig. 6 Cyclic voltammetric profiles of (a) AG, (b) HC/G-1 and (c) HC/ $\mathrm{G}-3$ electrodes at a scan rate of $0.5 \mathrm{mV} \mathrm{s}^{-1}$. corresponding to higher capacity. Moreover, the oxidation peaks gradually shift to a lower potential with the increase in loading amount of hard carbon, indicating reduced electrode polarization; this might suggest improved rate capability of the composites.

Fig. 7 illustrates the charge and discharge profiles of the first cycle for the electrodes in the voltage range of $0.005-2.0 \mathrm{~V}$ at a rate of $0.1 \mathrm{C}$ (corresponding to $35 \mathrm{~mA} \mathrm{~g}^{-1}$ ). Clearly, sloping region (from 1.25 to $0.15 \mathrm{~V}$ ) and plateau region (under $0.1 \mathrm{~V}$ ) corresponding to lithiation at amorphous carbons and graphitic carbons ${ }^{32}$ can be found for the composites. Moreover, with the increase in hard carbon ratio, the capacity contributed by the sloping region increases significantly, resulting in high total capacity. The galvanostatic test results of the initial cycle are summarized in Table 2. Notably, pristine AG exhibits charge/discharge capacity (CC/DC) of 356.8/381 $\mathrm{mA} \mathrm{h} \mathrm{g}^{-1}$ with initial coulombic efficiency (ICE) of 93.6\%, whereas HC/G-1 and HC/G-3 deliver CC/DC values of 365.9/401 and 374.7/ $418 \mathrm{~mA} \mathrm{~h} \mathrm{~g}^{-1}$ with ICE values of $91.2 \%$ and $89.6 \%$, respectively. This improvement in reversible capacity of the novel composites is mostly due to the in situ anchoring of hard carbons and the minor P-doped structures. ${ }^{33}$

Rate capability and cycling performance are also tested for $\mathrm{HC} / \mathrm{G}$ composites to reveal the relationship between anode composition and electrochemical performance. As shown by

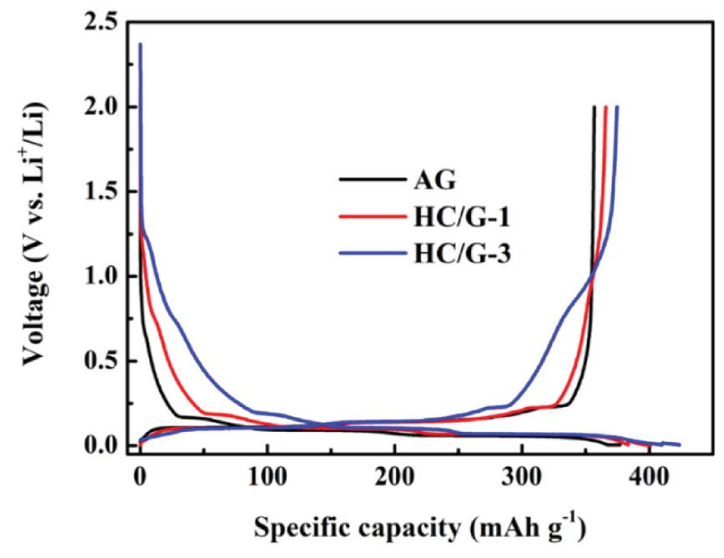

Fig. 7 Initial charge and discharge of $\mathrm{AG}, \mathrm{HC} / \mathrm{G}-1$ and $\mathrm{HC} / \mathrm{G}-3$ at current of $0.1 \mathrm{C}$.

Table 2 Electrochemical properties of the three samples

\begin{tabular}{|c|c|c|c|c|}
\hline & $\mathrm{RC}^{a}$ & $\mathrm{ICE}^{b}$ & $\mathrm{RE}^{c}{ }^{c}$ & $\mathrm{RE} 2^{d}$ \\
\hline Samples & $\left(\mathrm{mA} \mathrm{h} \mathrm{g}^{-1}\right)$ & $(\%)$ & $\%$ & $\%$ \\
\hline AG & 356.8 & 93.6 & 4.2 & 88.0 \\
\hline HC/G-1 & 365.9 & 91.2 & 12.1 & 97.1 \\
\hline HC/G-3 & 374.7 & 89.6 & 14.5 & 91.4 \\
\hline
\end{tabular}

${ }^{a}$ The first cycle discharge capacity. ${ }^{b}$ The initial coulombic efficiency. ${ }^{c}$ The retention ratio of charge capacity of the first cycle at $3 \mathrm{C}$ against the charge capacity of the second cycle at initial $0.1 \mathrm{C} .{ }^{d}$ The recovery ratio of the first cycle charge capacity of final $0.1 \mathrm{C}$ against the charge capacity of the second cycle at initial $0.1 \mathrm{C}$. 
the rate performance of $\mathrm{AG}$ and $\mathrm{HC} / \mathrm{G}$ composites from $0.1 \mathrm{C}$ to 3C in Fig. 8a, the capacities of $\mathrm{HC} / \mathrm{G}$ composites are higher than that of AG at all current densities, indicating that the introduction of hard carbon onto AG successfully improves its rate capability. This is mainly because hard carbon commonly exhibits larger interlayer spacing and some nanopores, which can provide more equivalent channels for lithium-ion diffusion. ${ }^{34}$ However, for the obtained composites, HC/G-1 shows higher charge capacities than $\mathrm{HC} / \mathrm{G}-3$ from 0.5 to $2 \mathrm{C}$; this is probably due to the greater amount of hard carbon in $\mathrm{HC} / \mathrm{G}-3$, which leads to poor conductivity. Under 3C, a higher charge capacity is found for $\mathrm{HC} / \mathrm{G}-3$, indicating that a high loading amount of hard carbon is critical to obtain high-rate performance of the $\mathrm{HC} / \mathrm{G}$ composite. As indicated by Table 2, capacity retention ratios (RE1) of 4.2, 12.1 and $14.5 \%$ are achieved at $3 \mathrm{C}$ for $\mathrm{AG}, \mathrm{HC} / \mathrm{G}-1$ and $\mathrm{HC} / \mathrm{G}-3$, respectively. When the C-rate is switched back to $0.1 \mathrm{C}$, the charge capacities return to 312.3 , 354.3 and $342.7 \mathrm{~mA} \mathrm{~h} \mathrm{~g}^{-1}$ with capacity recovery ratios (RE2) of 88.0, 97.1 and $91.4 \%$ for AG, HC/G-1 and HC/G-3, respectively, demonstrating good stability of the $\mathrm{HC} / \mathrm{G}-1$ electrode at various current densities.

Fig. $8 \mathrm{~b}$ shows the cycling performance of the obtained samples tested at 0.3C. During all the cycles, HC/G-1 and HC/G3 exhibit higher cycling reversible capacities and better cyclabilities than pristine AG. Additionally, both composites display a considerable capacity loss in the initial 20 cycles due to lithium consumption by active sites in the anchored hard carbon. However, in the following cycles, HC/G-1 exhibits a relatively stable charge/discharge process, whereas HC/G-3 suffers continuous capacity fading. After 100 cycles, HC/G-1 still exhibits capacity of $349 \mathrm{~mA} \mathrm{~h} \mathrm{~g}{ }^{-1}$, which is much higher than those of $\mathrm{HC} / \mathrm{G}-3\left(340 \mathrm{~mA} \mathrm{~h} \mathrm{~g}^{-1}\right)$ and AG $\left(325 \mathrm{~mA} \mathrm{~h} \mathrm{~g}^{-1}\right)$. The better cycling performance of HC/G-1 can be due to its proper HC-to-AG ratio, which is conducive to electrolyte infiltration; ${ }^{35}$ this can help improve ion-diffusion and maintain interfacial stability during cycling.

The dynamic processes of AG, HC/G-1 and HC/G-3 electrodes before cycling can be revealed by electrochemical impedance spectroscopy (EIS, as shown in Fig. 8c). The depressed semicircles in the high- and medium-frequency regions represent the total interfacial resistance from SEI $\left(R_{\mathrm{f}}\right)$ and the chargetransfer resistance $\left(R_{\mathrm{ct}}\right) .{ }^{36}$ The smaller the semicircle, the lower the total $R_{\mathrm{f}}$ and $R_{\mathrm{ct}}{ }^{37}$ The EIS curves are fitted by an equivalent circuit composed of "R(C(R))(C(RW))" using the ZSimpWin program (inset of Fig. 8c), and the fitting results are shown in Table 3. Among the samples, HC/G-1 has the lowest $R_{\mathrm{f}}$ and $R_{\text {ct }}$ values, which can verify its better rate performance mentioned above.

To further study the initial reaction kinetics of $\mathrm{HC} / \mathrm{G}, \mathrm{Li}^{+}$ diffusion coefficient $\left(D_{\mathrm{Li}^{+}}\right)$is calculated using the following equation: ${ }^{38}$

$$
D_{\mathrm{Li}^{+}}=R^{2} T^{2} / 2 A^{2} n^{4} F^{4} C_{\mathrm{Li}^{+}}{ }^{2} \delta^{2}
$$

Here, $R$ is the gas constant, $T$ is the absolute temperature, $A$ is the surface area of electrode, $n$ is the number of electrons per molecule, $F$ is the Faraday constant, $C_{\mathrm{Li}^{+}}$is the concentration of $\mathrm{Li}^{+}$, and $\delta$ is the Warburg coefficient. The real part of the impedance $\left(Z^{\prime}\right)$ is given by ${ }^{39,40}$ (a)

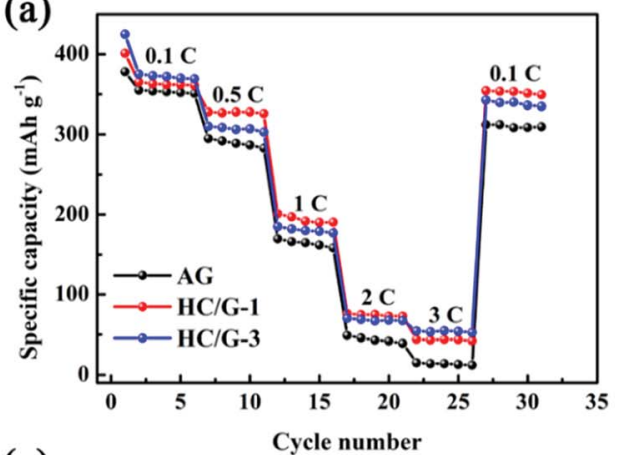

(c)

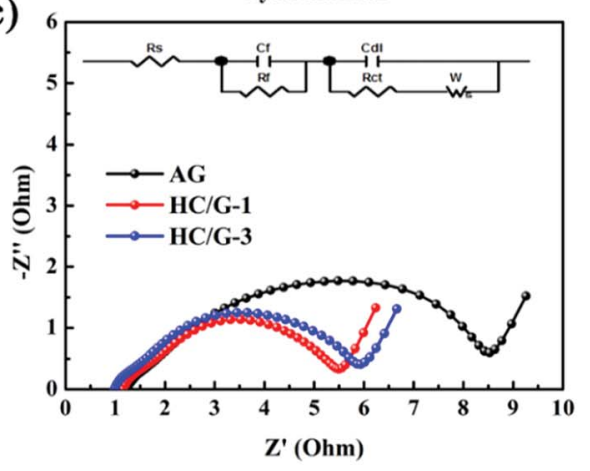

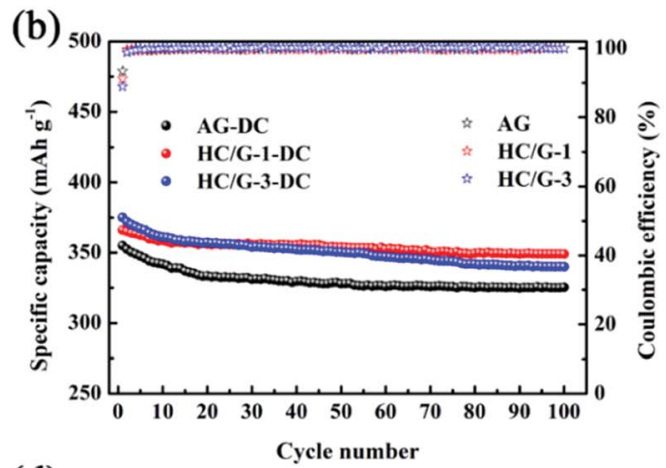

(d)

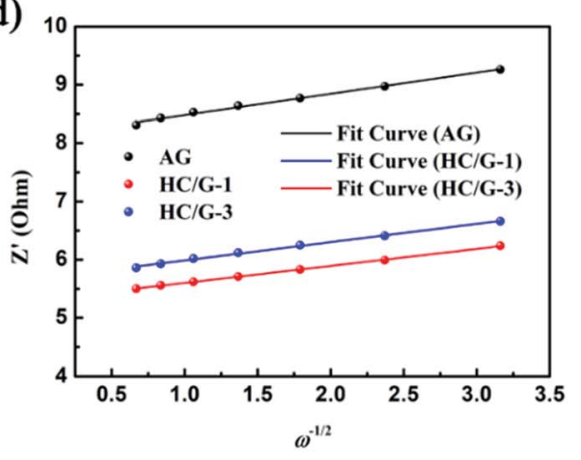

Fig. 8 Electrochemical performances of AG, HC/G-1 and HC/G-3 samples: (a) rate capability from 0.1C to 3C; (b) cycle performance at a current rate of $0.3 \mathrm{C}$; (c) Nyquist plots before cycling and (d) the relationship curves between $Z^{\prime}$ and $\omega^{-1 / 2}$ in the low-frequency region. 
Table 3 EIS parameters of the three samples

\begin{tabular}{lllll}
\hline Sample & $R_{\mathrm{f}}(\Omega)$ & $R_{\mathrm{ct}}(\Omega)$ & $\delta\left(\Omega \mathrm{s}^{-1 / 2}\right)$ & $D_{\mathrm{Li}^{+}}\left(\mathrm{cm}^{2} \mathrm{~s}^{-1}\right)$ \\
\hline AG & 1.8 & 4.0 & 0.381 & $1.03 \times 10^{-7}$ \\
HC/G-1 & 1.1 & 2.4 & 0.297 & $1.71 \times 10^{-7}$ \\
HC/G-3 & 1.3 & 2.6 & 0.321 & $1.47 \times 10^{-7}$
\end{tabular}

$$
Z^{\prime}=R_{\mathrm{c}}+R_{\mathrm{ct}}+\delta \omega^{-1 / 2}
$$

where $\omega$ is the angular frequency; both $R_{\mathrm{c}}$ and $R_{\mathrm{ct}}$ are the kinetic parameters independent of frequency and thus, $\delta$ is the slope of the plot of $Z^{\prime} v s$. the reciprocal square root of the lower angular frequencies $\left(\omega^{-1 / 2}\right)$. Based on the linear fitting of $Z^{\prime} v s . \omega^{-1 / 2}$ in the low-frequency region (Fig. $8 \mathrm{~d}$ ), the calculated average $D_{\mathrm{Li}^{+}}$ values of HC/G-1 $\left(1.71 \times 10^{-7} \mathrm{~cm}^{2} \mathrm{~s}^{-1}\right)$ and HC/G-3 $\left(1.47 \times 10^{-7}\right.$ $\left.\mathrm{cm}^{2} \mathrm{~s}^{-1}\right)$ are higher than that of pristine AG $\left(1.03 \times 10^{-7} \mathrm{~cm}^{2}\right.$ $\mathrm{s}^{-1}$ ). The increased $D_{\mathrm{Li}^{+}}$of the $\mathrm{HC} / \mathrm{G}$ composites suggests that the in situ anchoring of hard carbon onto the graphite surface can efficiently enhance $\mathrm{Li}^{+}$diffusivity in the pristine graphite. According to the superior electrochemical performance, $\mathrm{HC} / \mathrm{G}-1$ with an optimal HC-to-AG ratio can be a promising alternative anode for lithium-ion batteries.

\section{Conclusions}

Novel hard carbon/graphite composites are successfully synthesized by a facile in situ particle anchoring method. The obtained samples are evaluated in detail with the assistance of SEM, XRD, Raman and nitrogen adsorption and applied as high-performance graphitic anode materials for lithium-ion batteries. The results show that the specific surface area of the obtained composites gradually decreases with the increase in hard carbon amount. Among the samples tested in this study, HC/G-1 with an optimal HC-to-AG ratio displays the most favorable initial reversible capacity $\left(366 \mathrm{~mA} \mathrm{~h} \mathrm{~g}{ }^{-1}\right)$, cyclic capacity retention (349 $\mathrm{mA} \mathrm{h} \mathrm{g}^{-1}$ after 100 cycles at $0.3 \mathrm{C}$ ), and rate capability (capacity retention of $12.1 \%$ at $3 \mathrm{C}$ ). The improved electrochemical performance is due to the presence of a proper ratio of isotropic and porous hard carbon, which can offer fast transport channels for lithium ions and help electrolyte permeation, thus alleviating capacity fading during long cycling. In conclusion, this $\mathrm{HC} / \mathrm{G}$ composite has great potential to be an excellent candidate for anodes in high-energy and highpower-density lithium-ion batteries.

\section{Conflicts of interest}

There are no conflicts to declare.

\section{Acknowledgements}

This work was supported by China Ministry of Science and Technology (No. 2016YFB0100400).

\section{References}

1 F. Wang, W. Li, M. Hou, C. Li, Y. Wang and Y. Xia, J. Mater. Chem. A, 2015, 3, 1703-1708.

2 Y. Wang, X. Zheng, Q. Qu, G. Liu, V. S. Battglia and H. Zheng, Carbon, 2018, 132, 420-429.

3 J. Lu, Z. Chen, F. Pan, Y. Cui and K. Amine, Electrochemical Energy Reviews, 2018, 1, 1-19.

4 Y. J. Han, J. Kim, J. S. Yeo, J. C. An, I. P. Hong, K. Nakabayashi, J. Miyawaki, J. D. Jung and S. H. Yoon, Carbon, 2015, 94, 432-438.

5 C. Wan, H. Li, M. Wu and C. Zhao, J. Appl. Electrochem., 2009, 39, 1081-1086.

6 H. L. Zhang, S. H. Liu, F. Li, S. Bai, C. Liu, J. Tan and H. M. Cheng, Carbon, 2006, 44, 2212-2218.

7 B. Veeraraghavan, J. Paul, B. Haran and B. Popov, J. Power Sources, 2002, 109, 377-387.

8 J. S. Yeo, T. H. Park, M. H. Seo, J. Miyawaki, I. Mochida and S. H. Yoon, Int. J. Electrochem. Sci., 2013, 8, 1308-1315.

9 S. Y. Kim, J. Lee, B. H. Kim, Y. J. Kim, K. S. Yang and M. S. Park, ACS Appl. Mater. Interfaces, 2016, 8, 12109-12117. 10 Z. Yi, N. Lin, Y. Zhao, W. Wang, Y. Qian, Y. Zhu and Y. Qian, Energy Storage Mater., 2018, DOI: 10.1016/ j.ensm.2018.07.025.

11 Z. Yi, N. Lin, T. Xu and Y. Qian, Chem. Eng. J., 2018, 347, 214222.

12 A. Ulus, Y. Rosenberg, L. Burstein and E. Peled, J. Electrochem. Soc., 2002, 149, A635-A643.

13 J. Y. Lee, R. Zhang and Z. Liu, J. Power Sources, 2000, 90, 7075.

14 K. J. Kim, T. S. Lee, H. G. Kim, S. H. Lim and S. M. Lee, Electrochim. Acta, 2014, 135, 27-34.

15 Y. S. Ding, W. N. Li, S. Iaconetti, X. F. Shen, J. D. Carlo, F. S. Galasso and S. L. Suib, Surf. Coat. Technol., 2006, 200, 3041-3048.

16 C. Wang, H. Zhao, J. Wang and P. Lv, Ionics, 2013, 19, 221226.

17 H. Nozaki, K. Nagaoka, K. Hoshi, N. Ohta and M. Inagaki, J. Power Sources, 2009, 194, 486-493.

18 Y. Wang, S. Zuo, J. Yang and S. H. Yoon, Langmuir, 2017, 33, 3112-3122.

19 C. S. Wu, Y. L. Liu, Y. C. Chiu and Y. S. Chiu, Polym. Degrad. Stab., 2002, 78, 41-48.

20 N. Ohta, H. Nozaki, K. Nagaoka, K. Hoshi, T. Tojo, T. Sogabe and M. Inagaki, New Carbon Mater., 2002, 17, 61-63.

21 M. Yoshio, H. Wang, K. Fukuda, Y. Hara and Y. Adachi, J. Electrochem. Soc., 2000, 147, 1245-1250.

22 T. Zheng, J. S. Xue and J. R. Dahn, Chem. Mater., 1996, 8, 389-393.

23 Z. Q. Li, C. J. Lu, Z. P. Xia, Y. Zhou and Z. Luo, Carbon, 2007, 45, 1686-1695.

24 X. Jiang, H. C. Fang, P. Xiao, T. Liu, J. M. Zhu, Y. C. Wang, P. F. Liu and Y. Li, J. Alloys Compd., 2018, 744, 165-173.

25 H. Takagi, K. Maruyama, N. Yoshizawa, Y. Yamada and Y. Sato, Fuel, 2004, 83, 2427-2433. 
26 K. Hoshi, N. Ohta, K. Nagaoka, S. Bitoh, A. Yamanaka, H. Nozaki, T. Okuni and M. Inagaki, Tanso, 2009, 2009, 213-220.

27 L. G. Cançado, K. Takai, T. Enoki, M. Endo, Y. A. Kim, H. Mizusaki, N. L. Speziali, A. Jorio and M. A. Pimenta, Carbon, 2008, 46, 272-275.

28 D. A. Stevens and J. R. Dahn, J. Electrochem. Soc., 2001, 148, A803-A811.

29 M. J. Lázaro, L. Calvillo, E. G. Bordejé, R. Moliner, R. Juan and C. R. Ruiz, Microporous Mesoporous Mater., 2007, 103, 158-165.

30 H. Fujimoto, K. Tokumitsu, A. Mabuchi, N. Chinnasamy and T. Kasuh, J. Power Sources, 2010, 195, 7452-7456.

31 J. S. Park, J. Lee, J. W. Lee, K. J. Kim, Y. N. Jo, S. G. Woo and Y. J. Kim, Carbon, 2013, 62, 278-287.

32 R. Imamura, K. Matsui, S. Takeda, J. Ozaki and A. Oya, Carbon, 1999, 37, 261-267.
33 H. Tao, S. Du, F. Zhang, L. Xiong, Y. Zhang, H. Ma and X. L. Yang, ACS Appl. Mater. Interfaces, 2018, DOI: 10.1021/ acsami.8b11243.

34 J. Yang, X. Y. Zhou, J. Li, Y. I. Zou and J. J. Tang, Mater. Chem. Phys., 2012, 135, 445-450.

35 Y. Bai, Y. Liu, Y. Li, L. Ling, F. Wu and C. Wu, RSC Adv., 2017, 7, 5519-5527.

36 V. S. Kolosnitsyn, E. V. Kuzmina, E. V. Karaseva and S. E. Mochalov, J. Power Sources, 2011, 196, 1478-1482.

37 X. Wu, X. Yang, F. Zhang, L. Cai, L. Zhang and Z. Wen, Ceram. Int., 2017, 43, 9458-9464.

38 L. L. Zhang, H. B. Sun, X. L. Yang, Y. W. Wen, Y. H. Huang, M. Li, G. Peng, H. C. Tao, S. B. Ni and G. Liang, Electrochim. Acta, 2015, 152, 496-504.

39 L. C. Zou and C. Hunt, J. Electrochem. Soc., 2009, 156, 8-15. 40 X. L. Wu, Y. G. Guo, J. Su, J. W. Xiong, Y. L. Zhang and L. J. Wan, Adv. Energy Mater., 2013, 3, 1155-1160. 